\title{
THE METHOD OF SPECTRALLY SELECTIVE LASER MUELLER MATRIX POLARIMETRY FOR VERIFICATION OF ACUTE CORONARY INSUFFICIENCY
}

\author{
Oleh Vanchuliak ${ }^{1}$, Victor Bachinskiy ${ }^{2}$, Alexander Ushenko ${ }^{3}$
}

\begin{abstract}
INTRODUCTION: As the existing methods of evaluation of acute coronary insufficiency currently held is to some extent subjective. However, the specificity of forensic medicine requires objective methods. Thus, there is a necessity for objective methods of diagnosis for acute coronary insufficiency (ACI).
\end{abstract}

OBJECTIVES: The objective of this study is to establish the diagnostic possibilities of the laser method, Mueller matrix polarimetry, on wavelength $450 \mathrm{~nm}$ of autofluorescence, with the method of statistical analysis of native heart slices to provide an after-death diagnosis of acute coronary insufficiency.

METHODS: In our primary investigation we explored 69 human hearts with ACI, 69 with chronic ischemic heart disease, and 20 from corpses after violent deaths with short agonal period. The samples were studied with a Stokes polarimeter, which was modified to study laser induced autofluorescence. For comparison, histopathological studies were conducted by staining with hematoxylin, basic fuchsine, and picric acid. Data were analyzed statistically.

RESULTS: Coordinate and probabilistic distributions of Mueller matrix invariant $\mathbf{r}_{14}$ and $\mathbf{r}_{41}$ laser polarization autofluorescence samples of myocardium were established. A visual determination of ACI, using coordinate distributions or probabilistic distributions, is difficult and hence, we conducted a statistical analysis between pixels of the image. For optically active fluorophores of the myocardium we found a growth (1.5 times) kurtosis of $r_{41}$ value. Distribution of $r_{41}$ decreased 1.6 times in the case of ACI. After definition of balanced accuracy, we noted definition of asymmetry and kurtosis of invariant $r_{41}$ with $89 \%$ and $95 \%$ accuracy allowed a diagnosis of ACI.

CONCLUSION: Using the spectrally selective laser method, Mueller-matrix polarimetry, on wavelength 450 $\mathrm{nm}$ of autofluorescence with method of statistical analysis of native heart slices allows for diagnosis of ACI with high accuracy.

UDC Classification: 616-001/-009 DOI: http://dx.doi.org/10.12955/cbup.v4.836

Keywords: Diagnostic, ischemia, myocardium, autopsy.

\section{Introduction}

Ischemic heart disease has been shown as the underlying cause of sudden cardiac death (SCD) in $\sim 80 \%$ of victims in western societies. These SCDs, involving cardiac arrest, often occur with preceding symptoms of myocardial ischemia and are possibly accompanied with fresh thrombus when examined by coronary angiography or at autopsy (World Health Organization, 2004). However, as there are no witnesses and no significant signs at autopsy, reaching a conclusion about the presence of acute coronary syndrome or insufficiency (ACI) is difficult for a forensic expert (Hookana et al., 2014). Dettmeyer (2011) noted that, for histopathological verification, there are few signs in the case of myocardial infarction less than six hours after the moment of cardiac arrest to the moment of death. As the evaluation of existing diagnostic methods currently held is to some extent subjective and the specificity of forensic medicine requires objective methods. Thus, there is a necessity for objective methods of diagnosis of ACI.

\section{Literature Review}

According to Angelsky Ushenko, Ushenko, and Pishak (2007), there is a new and quickly developing scientific field, i.e., optical analysis of biological tissues. According to Tuchin (2002), analysis of polarization characteristics of scattered radiation is a way to qualitatively acquire new results about the

\footnotetext{
${ }^{1}$ Oleh Vanchuliak, Department of Forensic Medicine and Medical Law, Ukrainian State higher educational institution, Bucovinian State Medical University, Chernivtsi, Ukraine, wanchulyak@ gmail.com

${ }^{2}$ Victor Bachinskiy, Department of Forensic Medicine and Medical Law, Ukrainian State higher educational institution, Bucovinian State Medical University, Chernivtsi, Ukraine, vbachinskiy@ mail.ua

${ }^{3}$ Alexander Ushenko, Department of Correlation Optics and Spectroscopy of Ukrainian State higher educational institution, Yurii Fedkovych Chernivtsi National University, Yurii Fedkovych Chernivtsi National University, o.ushenko@chnu.edu.ua
} 
morphological and physiological state of biological tissues. Yao (1999) concluded that most biological tissues are characterized by optical anisotropy at both the microscopic and macroscopic levels, including the myocardium.

According to Yao and Wang (1999), the most complete properties of the myocardium can be studied by Mueller matrix analysis.

Nevertheless, Alfano et al. (1984) stated that human tissue contains complex endogenous fluorophores. Andersson-Engelsy, af Klinteberg, Svanberg, and Svanberg (1997) found that identifying autofluorescence of tissues opened an opportunity to label biochemical processes of cells and tissues, including ischemia. Thus, based on the combined use of methods for studying laser polarization and autofluorescence images, we endeavored to establish the presence of ACI. Subsequently, the objective of this study is to establish the diagnostic possibilities of the laser Muellermatrix polarimetry (wavelength $450 \mathrm{~nm}$ ) of autofluorescence combined with the method of statistical analysis of native heart slices to allow for after-death diagnosis of ACI.

\section{Data and Methodology}

We investigated 69 human hearts with ACI, 69 with chronic ischemic heart disease (CIHD), and 20 from corpses, after violent death, with short agonal period. This material was collected from 2010 to 2015 at the Chernivtsi Regional Bureau of Forensics, Ukraine.

Samples with a volume of $1-\mathrm{cm}^{3}$ were excised directly after organ collection. These were excised on a freezing microtome with a slice thickness of $30 \pm 5$ microns. The samples were then dried. The dried samples were then studied with a Stokes polarimeter, which was modified to study laser induced autofluorescence. For inciting autofluorescence in samples of infarction, we used the 'blue' solid-state semiconductor laser with a wavelength of $450 \mathrm{~nm}$ at $100 \mathrm{Mw}$ power. We used an area equal to $(1280 \times$ 960 pixels) of a CCD-camera (The Imaging Source DMK 41AU02.AS; monochrome 1/2 inch CCD, Sony ICX205AL progressive scan; resolution $1280 \times 960$; photosensitive area $7600 \times 6200 \mu \mathrm{m}$; sensitivity $0.05 \mathrm{~lx}$; dynamic range 8-bit). Next, all calculations were conducted on the personal computer program MATLAB. At the same time, histopathological studies were conducted by staining with hematoxylin, basic fuchsine, and picric acid.

\section{Results and Discussion}

Measurement results of the coordinate and statistical patterns in a set of Mueller matrix invariants of samples under conditions of ACI and CIHD for the spectral region of wavelength $450 \mathrm{~nm}$ are presented in Figure 1.

According to Andersson-Engelsy (1997), after transition from a reductive nicotinamide adenine nucleotide $\left(\mathrm{NADH}_{2}\right)$ to the oxidized (NAD) state, it loses an absorption band and the capacity for fluorescence. Because accumulation of $\mathrm{NADH}_{2}$ was observed during myocardial ischemia, one should expect an increase in modulation of secondary autofluorescence radiation. Indeed, during ACI there was intense autofluorescence (Figure $1 ; 4)$. However, there was sufficient proximity of histogram distributions of Mueller matrix invariant $r_{41}$ (Figure 2; $3 \& 4$ ), which determined the degree of crystallization of myocardial samples of both types.

A visual determination of ACI, using coordinate distributions or probabilistic distributions, was difficult and thus, we conducted a statistical analysis between the pixels of the image. The results are shown in Table 1.

The results confirmed experimentally the criteria of differentiation of ACI, i.e., normal heart and CIHD.

For optically active fluorophores of the myocardium, we found a growth (1.5 times) kurtosis of $r_{41}$ value. Distribution of $r_{41}$ decreased 1.6 times in the case of ACI.

Table 2 presents the balanced data accuracy for the spectrally selective laser Mueller matrix polarimetry autofluorescence at a wavelength of $450 \mathrm{~nm}$ for samples of the myocardium. Analysis of the balanced data, carried out on data according to Myers, demonstrates the sensitivity and specificity of spectrally selective laser Mueller matrix polarimetry autofluorescence of samples of the myocardium. The analysis conducted for the moments of both Mueller matrix invariants that were the most promising for verification ACI: asymmetry and kurtosis. 
Figure 1: Coordinate and probabilistic distributions of Mueller matrix invariant $r{ }_{14}$ laser polarization autofluorescence samples of myocardium

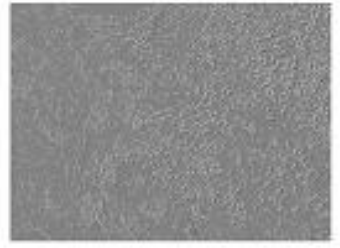

(1)

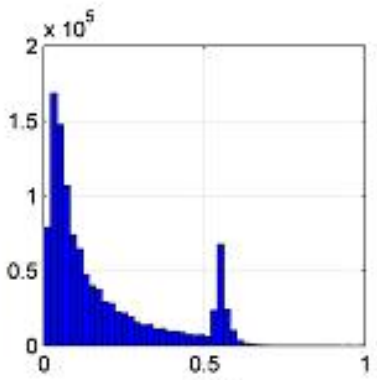

(3)

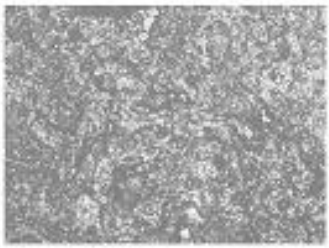

(2)

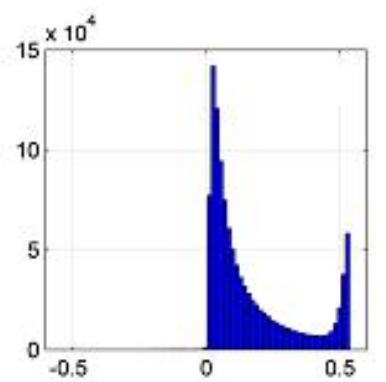

(4)

1: coordinate distribution of CIHD samples; 2: coordinate distribution of ACI samples; 3: probabilistic distribution of CIHD samples; 4: probabilistic distribution of ACI samples.

Source: Authors

Figure 2: Coordinate and probabilistic distributions of Mueller matrix invariant $\mathrm{r} 41$ laser polarization autofluorescence samples of myocardium

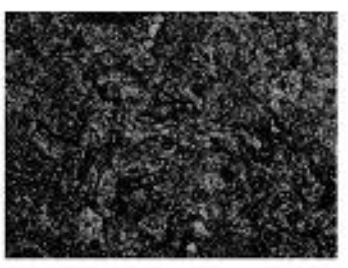

(1)

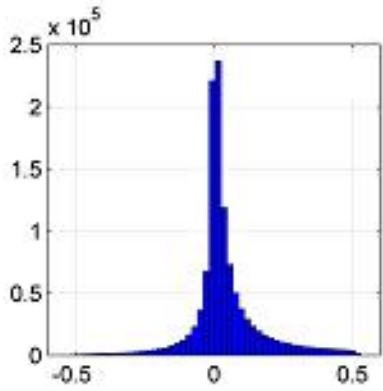

(3)

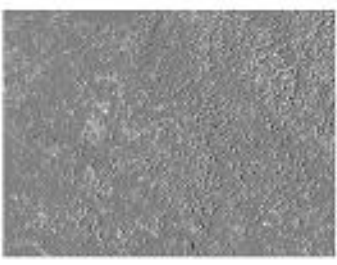

(2)



(4)

1: coordinate distribution of CIHD samples; 2: coordinate distribution of ACI samples; 3: probabilistic distribution of CIHD samples; 4: probabilistic distribution of ACI samples.

Source: Authors 
Table 1: Statistical moments for spectrally selective laser Mueller matrix polarimetry autofluorescence method

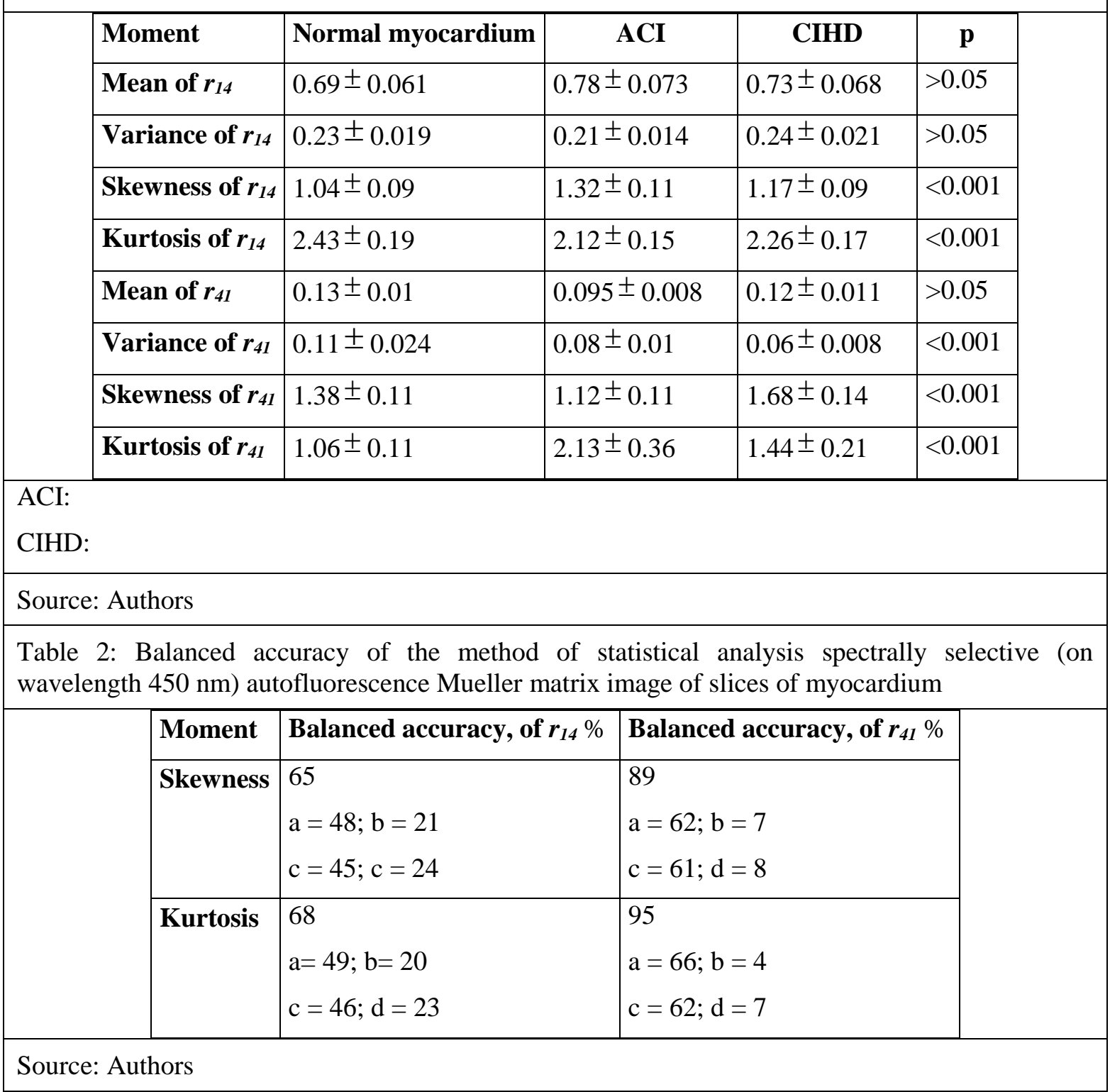

Definition of asymmetry and kurtosis of invariant $r_{14}$ with $89 \%$ and $95 \%$ accuracy allowed a diagnosis of ACI.

\section{Conclusion}

Using spectrally selective laser Mueller matrix polarimetry on wavelength $450 \mathrm{~nm}$ of autofluorescence with the method of statistical analysis of native heart slices allowed a diagnosis of ACI with high accuracy.

\section{Acknowledgements}

The authors thank the staff of the Department of Correlation Optics and Spectroscopy of Ukrainian State Higher Educational Institution, "Yurii Fedkovych Chernivtsi National University", for the opportunity to work in a laboratory.

\section{References}

Alfano, R. R., Tata, D. B., Cordero, J., Tomashefsky, P., Longo, F. W., \& Alfano, M. (1984). Laser induced fluorescence spectroscopy from native cancerous and normal tissue. Quantum Electronics, IEEE Journal of, 20(12), 1507-1511.

Andersson-Engels, S., af Klinteberg, C., Svanberg, K., \& Svanberg, S. (1997). In vivo fluorescence imaging for tissue diagnostics. Physics in medicine and biology, 42(5), 815. 
Angelsky, O. V., Ushenko, A. G., Ushenko, Y. A., \& Pishak, V. P. (2007). Statistical and fractal structure of biological tissue Mueller matrix images. Optical Correlation Techniques and Applications, 6616, 213-263.

Dettmeyer, R. (2011). Forensic histopathology: fundamentals and perspectives. Springer Science \& Business Media.

Hookana, E. K., Junttila, M. J., Kaikkonen, K. S., Kortelainen, M. L., Myerburg, R. J., \& Huikuri, H. V. (2014). Temporal Trends in the Characteristics of Autopsy-Verified Victims of Sudden Cardiac Death Without a Known Prior Cardiac Disease. Circulation, 130 (Suppl 2), A12141-A12141.

Tuchin, V. V. (2002). Handbook of optical biomedical diagnostics. Bellingham: SPIE Press, 1110.

World Health Organization (2004). International statistical classification of diseases and health related problems. The ICD10

Yao, G., \& Wang, L. V. (1999). Two-dimensional depth-resolved Mueller matrix characterization of biological tissue by optical coherence tomography. Optics Letters, 24(8), 537-539. 